\title{
Comparison of laminar and plug flow-fields on extraction performance in micro-channels
}

\author{
B. Malengier ${ }^{\mathrm{a}}$, J.L. Tamalapakula ${ }^{\mathrm{b}}$, S. Pushpavanam ${ }^{\mathrm{b}}$ \\ ${ }^{a}$ Department of Mathematical Analysis, Ghent University, Galglaan 2, 9000 Gent, Belgium \\ ${ }^{b}$ Department of Chemical Engineering, I.I.T Madras, 600036 India
}

\begin{abstract}
Several applications such as liquid-liquid extraction in micro-fluidic devices are concerned with the flow of two immiscible liquid phases. The stratified flow regime in these micro-channels has the inherent advantage that separation at the exit is efficient. In pressure driven flows in microchannels the flow profile is laminar and parabolic. This induces axial dispersion in the system which is known to reduce the efficiency of the process. In micro-channels electro-osmosis has been employed to overcome this drawback, leading to a flow profile that approaches the classical plug flow behaviour.

In this work fundamental features of the velocity profiles in stratified flows are analysed. First the flow between two rectangular infinite flat plates is discussed. The influence of physical properties, in particular viscosity of the two liquids, on the velocity profiles is determined symbolically. The flow-profiles are classified in the parameter space of physical properties (viscosity ratio) and operating conditions (flow-rate ratio). The mass transfer in these systems is compared with that found when the two liquids follow an ideal plug flow behaviour. A symbolic solution based on the method of separation of variables is proposed for concentration when the flow profile is a plug flow. It is found that the plug flow profile gives a poorer performance than the laminar profile. This is attributed to the lower velocity prevailing at the interface in the plug flow regime. To understand this more clearly the effect of changing the height of the interface was also analysed and it was found that under some conditions the ideal plug flow can give a better performance than the laminar reactor. The results of this work will aid the experimentalist establish guidelines to determine if introducing electro-osmosis in a Hagen-Poiseuille flow will be beneficial or detrimental in liquid-liquid extraction.
\end{abstract}

Keywords: Laminar flow, Convective transport, Mass transfer, Diffusion, Extraction, Mathematical modelling and simulation

\section{Introduction}

The physical effects which govern the behaviour of fluids flowing at the microscale and the macroscale are different. Surface tension, viscous effects, energy dissipation and capillary action begin to dominate system behaviour at the microscale. Microfluidics studies the behaviour of the fluids at the microscale induced by these effects. It helps exploit the behaviour at these scales for new uses by improving the efficiency of current processes.

Microfluidics results in process intensification through miniaturization in process industries. These systems are characterized by a dominance of viscous forces as compared to inertial forces; hence, low Reynolds numbers are encountered. Consequently, the flow regimes observed in these systems is laminar. Mixing in these micro-channels occurs primarily by molecular diffusion. The time needed for mixing by molecular diffusion is proportional to the square of the length of the

Email addresses: bm@cage.ugent.be (B. Malengier), jyothilathat@gmail.com (J.L. Tamalapakula), spush@iitm.ac.in (S. Pushpavanam ) 
diffusion path. The marked shortening of the diffusion path in a micro-channel results in relatively good mixing.

Multiphase liquid-liquid flows arise when two or more partially miscible or completely immiscible fluids are brought in contact and subjected to a pressure gradient. The resulting systems display different kinds of flow behaviour, e.g. droplet, slug or stratified flow. These regimes depend on the relative flow rates of the fluid phases involved, the resulting interaction between interfacial, and viscous forces and the wetting behaviour of the channel walls. Different liquid-liquid phase flow patterns in micro-channels have been experimentally analysed, see Dessimoz et al. [5]. Recently, Zhao et al. [16] studied mass transfer behaviour in a stainless steel micro-channel with a T-junction inlet under cross flow and opposing flow configurations. In Burns and Ramshaw [2] an immiscible liquid-liquid two phase flow system was analysed and the system was found to show stratified flow with a planar interface for a wide range of operating conditions. It was proposed that the slow flows provided opportunities for carrying out reactions and separations. In a later work the slug flow behaviour in liquid-liquid systems was analysed, see Burns and Ramshaw [3]. It was found that slug flow behaviour had a significant effect on the mass transfer rate of acetic acid from kerosene to the aqueous phase. They studied how the vortex motion within a slug could improve mass transfer across the interface. It was suggested that this could be exploited to control fast reactions which are mass transfer limited. These reactions include nitration, hydrogenation, sulfonation and oxidation of organic compounds. The slug flow regime is commonly encountered and has an important advantage that it facilitates rapid mass transfer as the surface area to volume ratio is significantly high. The biphasic hydrolysis of p-nitrophenylacetate was studied under stratified and segmented flow conditions. The effect of sonication and phase transfer catalysis in this system was analysed in Ahmed-Omer et al. [1]. The mass transfer rate also depends on the presence of a film outside the non wetting phase which is determined by the capillary number, see Taha and Cui [13], Dessimoz et al. [5].

In the context of mass transfer across membranes Guo and Ho [8] have analysed an symbolic solution based on separation of variables for co-current and counter-current flows in an annulus. The eigenfunctions were calculated using a power series expansion. The velocity fields in the two chambers were decoupled as they were separated by a porous membrane. Electro-osmotic flows in micro channels have been frequently proposed as being useful for overcoming dispersion effects. This causes the velocity profile in the micro-channel to remain almost uniform except for a small region near the walls. This helps the flow-field evolve to a plug-flow regime in the micro-channel as opposed to the parabolic laminar profile. Liu et al. [9] and Gao et al. [7] have studied the electro-osmotic flow in a rectangular channel when one fluid is conducting and the other is non conducting as it usually the case in extraction. The flow-profiles were obtained symbolically for the two fluids when the non conducting fluid was dragged by the viscous force of the conducting fluid. Wang et al. [15] have studied experimentally as well as theoretically how the interface level can be controlled in a two liquid system under the influence of a pressure drop and an electric field applied to one of the fluids. Their results indicate that the flow profiles in the two fluids can be approximated as plug-flows with a jump discontinuity at the interface for some operating conditions.

Recently studies have focussed on liquid-liquid extraction in the stratified flow regime in the micro-channels. The stratified flow pattern can be exploited to facilitate complete separation at the channel exit. The extraction of vanillin dissolved in water using toluene in micro-structured devices made of Polydimethylsiloxane (PDMS) was studied by Fries et al. [6]. Here the performance of segmented and stratified flow regimes were compared. Laser-induced fluorescence (LIF) and microscopic particle image velocimetry (microPIV) measurements showed a laminar profile for stratified flow, whereas vortices in the slug were detected for segmented flow. The influence of channel width and therefore, the surface-to-volume ratio was investigated for stratified flow. There was a significant enhancement of mass transfer with decrease in the channel cross-sectional area for the stratified flow regime.

Three different fluid-flow patterns in a Y-shaped micro-channel, contact or stratified flow, segmented flow and emulsification were investigated in Okubo et al. [11]. Here a one-dimensional model for extraction assuming the interface to be at the centre of the channel was used to compare 


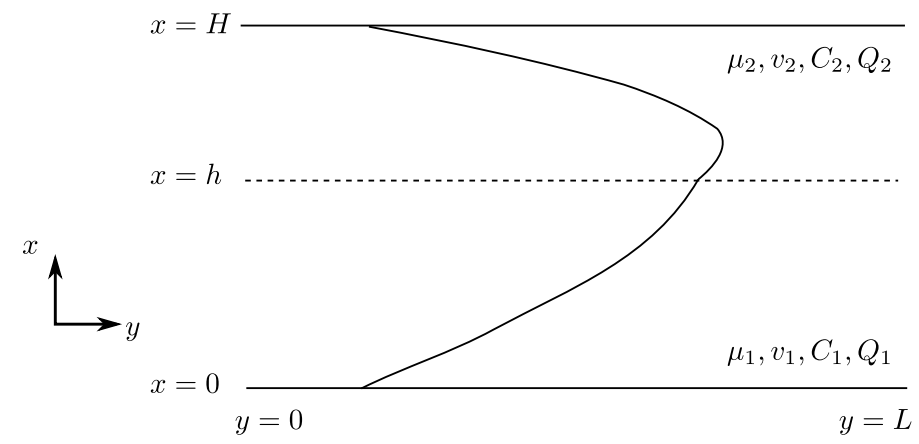

Figure 1: Schematic picture of the stratified flow system showing all variables. Velocity profile is for $\mu_{1}>\mu_{2}$, $\Delta P<0$.

the model predictions with the experimental behaviour. A two-dimensional flow-field taking into account the effect of the interface not being at the centre was analysed by Žnidaršič-Plazl and Plazl [14]. They compare their model predictions with experimental results on steroid extraction.

Modelling work on extraction in micro-channels has been mostly confined so far to preliminary studies. In Malengier et al. [10] the authors developed a model for stratified flow, indicating how performance can be optimized. In this work the extraction of a system is analysed when the flow-rates of the two fluids is fixed. The interface height is dependent on the viscosities and is determined from the flow field. A closed form symbolic solution for the concentration profile is established for the case when the velocity fields are uniform in each fluid. As our system is linear we view the governing differential equation and boundary conditions in the framework of linear operator theory. An inner product in which the system (differential operator subject to the boundary conditions) is self-adjoint is used to find the solution in the form of a series expansion. This is used to determine the mass transfer behaviour. For the laminar flow a numerical solution based on the method of lines is used to determine the effect of different parameters on extraction efficiency for flow between parallel plates. The mass transfer performance of laminar and plug flow behaviour are compared under different conditions. These results are extended to the case of a rectangular micro channel using the finite volume method. Experimentally the electro-osmotic effect can be used to vary and control the interface height and it is shown that under some conditions the plug flow behaviour will be better than the laminar behaviour. A physical basis for the results obtained is presented. It is generally believed that electro-osmotic flow would improve the performance of the micro-channel. This work provides guidelines to decide whether electro-osmosis is necessary to improve the performance of extraction in stratified flows.

\section{Hydrodynamic behaviour}

First, the stratified flow of two liquid phases is analysed between two infinite horizontal plates extending to infinity in two directions. The distance between the plates (along the $x$-direction) is taken as $H$ and the liquid-liquid interface is at distance $h$ from the lower plate. The flow is assumed to be in the $y$-direction the plates extending to infinity in the $z$-direction. Fig. 1 shows a schematic of the set up along with the axis. The velocity profile is obtained assuming the flow to be steady, fully developed and the liquids to be incompressible. The velocity profiles of the system are governed by the equation

$$
\begin{array}{ll}
\frac{\partial p}{\partial y}=\mu_{1} \frac{\partial^{2} v_{1}}{\partial x^{2}} & \text { for } 0<x<h, \\
\frac{\partial p}{\partial y}=\mu_{2} \frac{\partial^{2} v_{2}}{\partial x^{2}} & \text { for } \quad h<x<H .
\end{array}
$$


These equations are subject to the conditions of no slip at the walls and continuity of velocity and shear stress at the interface which is located at $h$. So

$$
\begin{aligned}
v_{1} & =0 & & \text { for } x=0, \\
v_{1} & =v_{2} & & \text { for } x=h, \\
\mu_{1} \frac{\partial v_{1}}{\partial x} & =\mu_{2} \frac{\partial v_{2}}{\partial x} & & \text { for } x=h, \\
v_{2} & =0 & & \text { for } x=H .
\end{aligned}
$$

In the above the subscript 1 and 2 are used to denote the fluid in the first and second region respectively. The solutions to the above equation yield the velocity profiles of the two liquids as

$$
\begin{cases}v_{1}(x)=-\frac{x\left(h^{2}\left(\mu_{2}-\mu_{1}\right)-x H \mu_{1}+H^{2} \mu_{1}-x h\left(\mu_{2}-\mu_{1}\right)\right)}{2\left(h\left(\mu_{2}-\mu_{1}\right)+H \mu_{1}\right) \mu_{1}} \nabla P & \text { for } 0<x<h, \\ v_{2}(x)=-\frac{(H-x)\left(H h\left(\mu_{2}-\mu_{1}\right)+x H \mu_{1}-h^{2}\left(\mu_{2}-\mu_{1}\right)+x h\left(\mu_{2}-\mu_{1}\right)\right)}{2\left(h\left(\mu_{2}-\mu_{1}\right)+H \mu_{1}\right) \mu_{2}} \nabla P & \text { for } h<x<H .\end{cases}
$$

Here the imposed pressure gradient is denoted as $\nabla P$. The flow behaviour is hence similar to the Hagen-Poiseuille flow (parabolic in shape) where both the fluids are subject to the same pressure drop. The velocity field is continuous at the interface $h$ but its derivatives are discontinuous, Fig. 1. From the point of view of the experimentalist the flow rates $Q_{1}$ and $Q_{2}$ are fixed. They are given by

$$
\left\{\begin{aligned}
Q_{1} & =\int_{0}^{h} v_{1}(x) d x \\
& =-\frac{h^{2}\left(h^{2} \mu_{2}+\mu_{1}\left(4 H^{2}-(H+h)^{2}\right)\right)}{12 \mu_{1}\left(\mu_{1}(H-h)+\mu_{2} h\right)} \nabla P, \\
Q_{2} & =\int_{h}^{H} v_{2}(x) d x \\
& =-\frac{(h-H)^{2}\left(\mu_{1}(h-H)^{2}-h \mu_{2}(h-4 H)\right)}{12 \mu_{2}\left(\mu_{1}(H-h)+\mu_{2} h\right)} \nabla P .
\end{aligned}\right.
$$

These equations are used to determine the pressure drop $\nabla P$ and the height $h$ of the interface for a given combination of flow-rates and fluids. Alternatively, if the pressure drop and height of interface are specified, the velocity profiles in each liquid layer can be found and from this the flow rates can be determined. The velocity profile is parabolic in shape and hence it has a maximum. This can lie in either of the two liquids. To determine the operating parameters for which the maximum lies in one of the two liquids, the critical condition at which the maxima lies at the interface of the two liquids is found out by setting the derivative of the velocity equal to zero at the interface for each of the velocity expressions. This gives us the condition

$$
\frac{h_{\text {crit }}}{H}=\frac{1}{1+\sqrt{r}}, \quad r=\frac{\mu_{2}}{\mu_{1}}, \quad 0<h_{\text {crit }}<H,
$$

where $r$ is the ratio of the two viscosities. For a given combination of fluids (or viscosity of the fluids) we see that the interface height when the maximum of the velocity profile lies at the interface depends only on the viscosity ratio. The ratio of the flow-rates of the two fluids when the maximum of the velocity profile is at the interface can be found out from the corresponding velocity profiles,

$$
\frac{Q_{1}}{Q_{2}}=\frac{1}{\sqrt{r}}
$$

This ratio is also dependent only on $r$ and in particular the pressure drop does not influence the ratio of the flow-rates, as it effects both the flow-rates equally in a linear manner. A plot of the critical flow-rate ratio $Q_{1} / Q_{2}$ on $1 / r$ is shown in Fig. 2. Along the curve the maximum of the velocity profile lies on the interface. It is seen that the curve divides the first quadrant (the region of physically relevant space) into two regions. In region I the maximum lies in the first fluid $(0 \leq x \leq h)$ while in region II the maxima lies in the second fluid $(h \leq x \leq H)$. For a given $r$ we can hence choose two different values of $Q_{1} / Q_{2}$ one above and below the curve and compare the mass transfer characteristics. This would correspond to a situation when the same two fluids are chosen but the relative flow rates of the two fluids are different. Similarly for a given $Q_{1} / Q_{2}$ we 


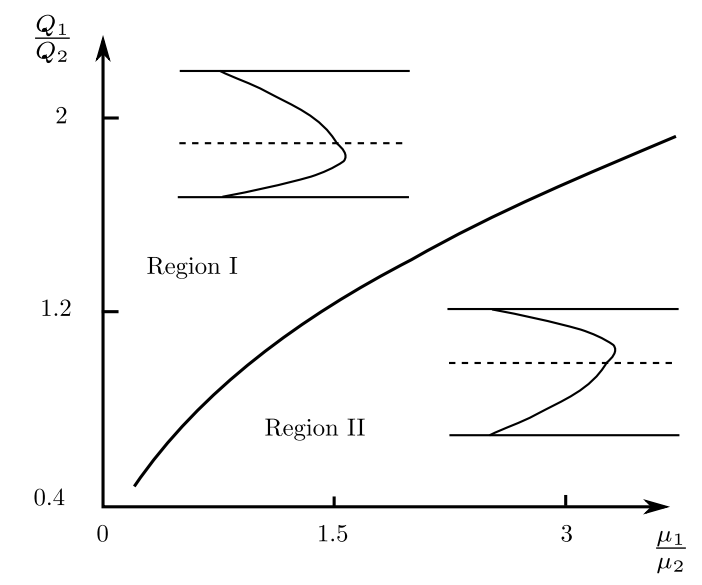

Figure 2: Dependence of the critical flow rate ratio $Q_{1} / Q_{2}$ when the maximum occurs at the interface on the ratio of the viscosities $\mu_{1} / \mu_{2}$. Velocity profiles in each region are shown.

could choose two different values of $r$ such that we are in the two regions and compare the mass transfer characteristics. This corresponds to a situation when the flow-rates of the two fluids are the same but the viscosity ratio of the two fluids are different. Any differences in the mass transfer behaviour in the two systems can be attributed to dispersion effects arising from the shapes of the velocity profiles.

\section{Mass transfer in extraction}

The mass transfer in a co-current stratified flow of a liquid-liquid extraction system in a microchannel is now analysed. Here we consider the flow of a solute in the first fluid which is being extracted by the second fluid. The concentration in fluid 1, respectively 2 is represented by $C_{1}$, respectively $C_{2}$. Considering steady-state operation with convection in the $y$-direction and diffusion in the $x$-direction we obtain the equations which govern the behaviour of the system as

$$
\begin{array}{ll}
v_{1} \partial_{y} C_{1}=D_{1} \partial_{x x} C_{1} & \text { for } \quad 0<x<h, \\
v_{2} \partial_{y} C_{2}=D_{2} \partial_{x x} C_{2} & \text { for } h<x<H .
\end{array}
$$

At the interface we have,

$$
\begin{aligned}
C_{1} & =K C_{2}, & & \text { for } x=h, \\
-D_{1} \partial_{x} C_{1} & =-D_{2} \partial_{x} C_{2}, & & \text { for } x=h,
\end{aligned}
$$

at the walls we have

$$
\begin{array}{ll}
\partial_{x} C_{1}=0, & \text { for } x=0, y>0 \\
\partial_{x} C_{2}=0, & \text { for } x=H, y>0,
\end{array}
$$

and at the inlet $y=0$ we have

$$
\begin{array}{ll}
C_{1}=C^{\text {in }} & \text { for } \quad 0<x<h, y=0 \\
C_{2}=0 & \text { for } h<x<H, y=0 .
\end{array}
$$

The film interface conditions result in a discontinuous concentration profile, while keeping the mass flux continuous. If $K>1, C_{2}$ remains below the value of $C_{1}$ at the interface. When $K<1$ the reverse is true and the second fluid extracts the solvent out of the first strongly. Here the concentration $C_{1}$ is depleted at the interface so as to build up a larger $C_{2}$ concentration. In our computations we use $C^{\text {in }}=1 \mathrm{~mol} / \mathrm{m}^{3}$. 


\section{Plug Flow Regime}

The convection diffusion equation can be solved symbolically and elegantly under the assumptions of (i) the Plug Flow Regime (PFR) which consists of a uniform velocity in the two fluids ( $v_{1}$ and $v_{2}$ are constant), and (ii) a constant transverse diffusion coefficient $\left(D_{1}\right.$ and $\left.D_{2}\right)$. We start with non dimensionalizing the equations with respect to their characteristic lengths and initial concentrations,

$$
x^{\star}=\frac{x}{H}, \quad y^{\star}=\frac{y}{L}, \quad h^{\star}=\frac{h}{H}, \quad \text { and } \quad C_{i}^{\star}=\frac{C_{i}}{C^{\text {in }}},
$$

which gives the dimensionless form as

$$
\partial_{y} C_{i}=\frac{1}{\mathrm{Pe}_{i}} \partial_{x x} C_{i}, \quad \text { where } \quad \mathrm{Pe}_{i}=\frac{v_{i} H^{2}}{D_{i} L}, i=1,2 .
$$

For simplicity, we have dropped and will drop the superscript $\star$ from now on. We seek the solution $C_{i}(x, y)$ in the form $g_{i}(y) f_{i}(x)$. Substituting this in $(7)$ gives

$$
\frac{g_{i}^{\prime}(y)}{g_{i}(y)}=\frac{1}{\mathrm{Pe}_{i}} \frac{f_{i}^{\prime \prime}(x)}{f_{i}(x)}=-\lambda^{2},
$$

or

$$
\begin{aligned}
f_{i}^{\prime \prime}(x)+\lambda^{2} \mathrm{Pe}_{i} f_{i}(x) & =0, \\
g_{i}^{\prime}(y)+\lambda^{2} g_{i}(y) & =0 .
\end{aligned}
$$

This results in an eigenvalue problem in the $x$ direction whose solution is

$$
\begin{array}{ll}
f_{1}(x)=a \sin \left(\lambda \sqrt{\mathrm{Pe}_{1}} x\right)+b \cos \left(\lambda \sqrt{\mathrm{Pe}_{1}} x\right), & 0<x<h, \\
f_{2}(x)=c \sin \left(\lambda \sqrt{\mathrm{Pe}_{2}}(1-x)\right)+d \cos \left(\lambda \sqrt{\mathrm{Pe}_{2}}(1-x)\right), & h<x<1 .
\end{array}
$$

The boundary conditions at $x=0,1$ yield $a=c=0$. At $x=h$, the boundary condition $C_{1}=K C_{2}$ implies

$$
b \cos \left(\lambda \sqrt{\mathrm{Pe}_{1}} h\right)=K d \cos \left(\lambda \sqrt{\mathrm{Pe}_{2}}(1-h)\right),
$$

while $D_{1} \partial_{x} C_{1}=D_{2} \partial_{x} C_{2}$ implies

$$
-b D_{1} \lambda \sqrt{\mathrm{Pe}_{1}} \sin \left(\lambda \sqrt{\mathrm{Pe}_{1}} h\right)=d D_{2} \lambda \sqrt{\mathrm{Pe}_{2}} \sin \left(\lambda \sqrt{\mathrm{Pe}_{2}}(1-h)\right) .
$$

This yields the characteristic equation which determines the eigenvalues $\lambda$ as the solution to

$$
\sqrt{\mathrm{Pe}_{2}} \cos \left(\lambda \sqrt{\mathrm{Pe}_{1}} h\right) \sin \left(\lambda \sqrt{\mathrm{Pe}_{2}}(1-h)\right)+K \beta \sqrt{\mathrm{Pe}_{1}} \sin \left(\lambda \sqrt{\mathrm{Pe}_{1}} h\right) \cos \left(\lambda \sqrt{\mathrm{Pe}_{2}}(1-h)\right)=0,
$$

where $\beta=D_{1} / D_{2}$. The eigenfunctions corresponding to the $n$th eigenvalue is

$$
f^{n}(x)= \begin{cases}f_{1}^{n}(x)=b_{n} \cos \left(\lambda_{n} \sqrt{\mathrm{Pe}_{1}} x\right), & 0<x<h \\ f_{2}^{n}(x)=d_{n} \cos \left(\lambda_{n} \sqrt{\mathrm{Pe}_{2}}(1-x)\right), & h<x<1 .\end{cases}
$$

It has been shown in [12] that this system is self-adjoint in the inner product (when the velocity in the two fluids are equal $v_{1}=v_{2}$ )

$$
\left\langle f^{n}, f^{m}\right\rangle=\int_{0}^{h} f_{1}^{n} f_{1}^{m} d x+K \int_{h}^{1} f_{2}^{n} f_{2}^{m} d x .
$$

The eigenfunctions are normalized with respect to this inner product and the constants $b_{n}, d_{n}$ are obtained as

$$
\begin{aligned}
b_{n}=\left[\frac{2 \lambda_{n} \sqrt{\mathrm{Pe}_{1}} h}{4}+\sin \left(2 \lambda_{n} \sqrt{\mathrm{Pe}_{1}} h\right)\right. \\
\left.+K \frac{\beta^{2} P_{1} \sin ^{2}\left(\lambda_{n} \sqrt{\mathrm{Pe}_{1}} h\right)}{\mathrm{Pe}_{2} \sin ^{2}\left(\lambda_{n} \sqrt{\mathrm{Pe}_{2}}(1-h)\right)} \frac{2(1-h) \lambda_{n} \sqrt{\mathrm{Pe}_{2}}+\sin \left(2 \lambda_{n} \sqrt{\mathrm{Pe}_{2}}(1-h)\right)}{4 \lambda_{n} \sqrt{\mathrm{Pe}_{2}}}\right]^{-\frac{1}{2}},
\end{aligned}
$$




$$
d_{n}=-b_{n} \frac{\beta \sqrt{\mathrm{Pe}_{1}} \sin \left(\lambda_{n} \sqrt{\mathrm{Pe}_{1}} h\right)}{\sqrt{\mathrm{Pe}_{2}} \sin \left(\lambda_{n} \sqrt{\mathrm{Pe}_{2}}(1-h)\right)} .
$$

The solution for the $y$ dependency is $g_{n}(y)=k_{n} \exp \left(-\lambda_{n}^{2} y\right)$.

For co-current extraction the initial condition is $C_{1}=1$ for $0<x<h$ and $C_{2}=0$ for $h<x<1$. The coefficient $k_{n}$ can be obtained from the initial condition as

$$
k_{n}=b_{n} \frac{\sin \left(\lambda_{n} \sqrt{\mathrm{Pe}_{1}} h\right)}{\lambda_{n} \sqrt{\mathrm{Pe}_{1}}} .
$$

Since the boundary conditions are homogeneous Neumann conditions in the $x$-direction, $\lambda=0$ is also an eigenvalue, which corresponds to $n=1$. The eigenfunction corresponding to this is the equilibrium solution and is given by

$$
C^{\mathrm{eq}}(x, y)=\left\{\begin{array}{ll}
1 \\
1 / K
\end{array}\right\} \times \frac{h}{h+\frac{(1-h)}{K}}, \quad \begin{aligned}
& 0<x<h, \\
& h<x<1 .
\end{aligned}
$$

The complete solution to the convection diffusion equation is hence

$$
C(x, y)=\left\{\begin{array}{l}
1 \\
1 / K
\end{array}\right\} \times \frac{h}{h+\frac{(1-h)}{K}}+\sum_{n=2}^{\infty} b_{n} \frac{\sin \left(\lambda_{n} \sqrt{\mathrm{Pe}_{1}} h\right)}{\lambda_{n} \sqrt{\mathrm{Pe}_{1}}} \exp \left(-\lambda_{n}^{2} y\right) f^{n}(x), \quad \begin{array}{ll}
0<x<h, \\
h<x<1 .
\end{array}
$$

It was found that the above solution converged to the concentration profile when 50 terms are taken. This implies that (10) is to be solved for the first 50 roots. Since the roots are computed numerically care has to be taken to ensure that the roots are arranged in ascending order, there is no repetition of roots and no roots are missed out. This symbolic solution is used to validate the numerical code based on a second order finite difference scheme in space and method of lines. The numerical method was used to determine the behaviour of the laminar flow regime.

\section{Stratified flow in rectangular channels}

The results obtained up to now are valid for the case of the 1D channel i.e., stratified flow in a narrow gap between two large flat plates. Now, we extend this to stratified flow in a channel with a rectangular cross-section. Here the flow equations are described by

$$
\begin{array}{ll}
\nabla P=\mu_{1} \Delta v_{1}(x, z) & \text { for } 0<x<h, 0<z<W, \\
\nabla P=\mu_{2} \Delta v_{2}(x, z) & \text { for } h<x<H, 0<z<W .
\end{array}
$$

In this we assume the interface to be at a fixed position $h, \forall z \in(0, W)$. This is justified in the limit of low interfacial tensions.

To determine the flow in a rectangular channel of width $W$ and height $H$ one can exploit the fact that (14) is a linear elliptic non-homogeneous partial differential equation of the Poisson type. Using the method of full eigenfunction expansion, we obtain,

$$
\begin{aligned}
& v_{1}=\sum_{n=1}^{\infty} \sin \frac{n \pi z}{W}\left(a_{1, n} \sinh \frac{n \pi x}{W}+a_{2, n} \cosh \frac{n \pi x}{W}+1\right) a_{3, n} \\
& v_{2}=\sum_{n=1}^{\infty} \sin \frac{n \pi z}{W}\left(b_{1, n} \sinh \frac{n \pi x}{W}+b_{2, n} \cosh \frac{n \pi x}{W}+1\right) b_{3, n},
\end{aligned}
$$

The coefficients $a_{3, n}, b_{3, n}$ can be obtained by requiring that they satisfy (14). This gives

$$
\begin{aligned}
a_{3, n} & =-2 \frac{W^{2}}{\mu_{1} n^{3} \pi^{3}} \nabla P\left(1-(-1)^{n}\right) \\
b_{3, n} & =\frac{\mu_{1}}{\mu_{2}} a_{3, n}
\end{aligned}
$$




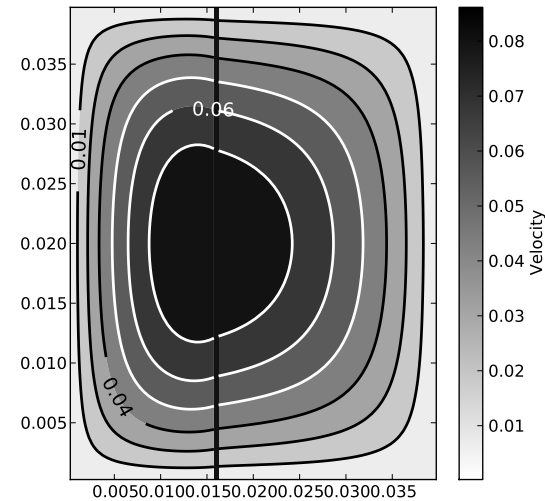

(a)

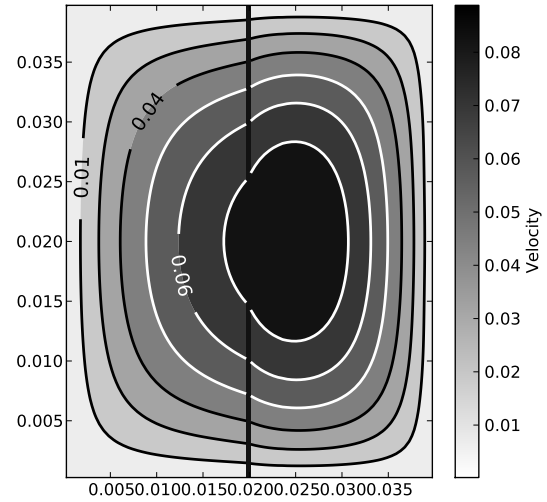

(b)

Figure 3: Velocity contours in a $0.04 \times 0.04$ square micro-channel for $Q_{1}=0.2766 \times 10^{-10} \mathrm{~m}^{3} / \mathrm{s}, Q_{2}=0.375 \times 10^{-10}$ $\mathrm{m}^{3} / \mathrm{s}$, (a) $\mu_{1}=0.001 \mathrm{Pas}, \mu_{2}=0.002 \mathrm{Pas}, h / H=0.4 \nabla P=-110 \mathrm{~Pa} / \mathrm{m}$. (b) $\mu_{1}=0.001 \mathrm{Pas}, \mu_{2}=0.0005 \mathrm{Pas}$, $h / H=0.498, \nabla P=-51.63 \mathrm{~Pa} / \mathrm{m}$, vertical black line is the interface.

Next, imposing the boundary conditions on the velocities gives expressions for the unknown coefficients $a_{1, n}, b_{1, n}, a_{2, n}, b_{2, n}$. For an accurate solution of the velocity field, one must consider a sufficiently large number of terms in the infinite sum. However, $\sinh (x)$ tends to $\cosh (x)$ for large values of $x$. This can lead to large numerical errors, and some of the terms become numerically unbounded. This is addressed in the limit of large values of the index of summations by using $a_{1, n}=-a_{2, n}$ and $b_{1, n}=-b_{2, n}$. The analytical expression is used to plot contours of the velocity field shown in Fig. 3. It was found that 60 terms were sufficient to obtain a converged solution (taking additional terms terms do not affect the solution anymore on the chosen grid). The contours were plotted by considering 60 terms in the series expansion. This was found to be sufficient to get smooth profiles. The flow rates corresponding to this flow field can be computed numerically. The species balance equations are solved using a finite volume approach for this geometry.

\section{Results and Discussion}

In this work our focus is on stratified flow behavior. The advantages of this flow stem from the ease of separation of the two streams at the channel exit. This has been experimentally exploited by Okubo et al. [11], Fries et al. [6] and by Žnidaršič-Plazl and Plazl [14]. Hence the stratified flow behavior discussed in this work is of practical relevance. The operating conditions i.e., flow rate combinations under which this flow-regime and the other commonly observed slug-flow regime is observed has been reported in Cherlo et al. [4].

In order to compare the results of our simulations and to be consistent with the literature, and evaluate the performance of a specific micro-channel set-up, we introduce some characteristic quantities. These are now defined.

\subsection{Characteristic quantities}

The first is the efficiency $E$, defined in terms of the mixed cup concentrations as

$$
E=\frac{C_{2}(L)-C_{2}(0)}{C_{2}^{\mathrm{eq}}-C_{2}(0)}
$$

where $C_{2}^{\mathrm{eq}}$ is the equilibrium outflow concentration in the second region, and typically $C_{2}(0)=0$. Furthermore, the mixed cup concentration is given by $C_{i}(y)=\iint v_{i} C_{i}(x, y, z) d x d z$. This is 


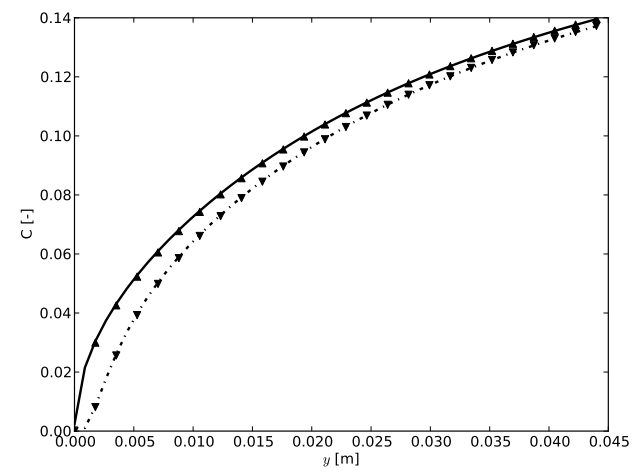

Figure 4: Comparison of the symbolic solution with 50 terms and the numerical computation in PFR. Here $H=$ $410^{-4} \mathrm{~m}, h=210^{-4} \mathrm{~m}, Q_{1}=Q_{2}=14.2857 \times 10^{-6} \mathrm{~m}^{2} / \mathrm{s}, K=4.14, D_{1}=7.4 \times 10^{-9} \mathrm{~m}^{2} / \mathrm{s}, D_{2}=5.64 \times 10^{-8}$ $\mathrm{m}^{2} / \mathrm{s}$. Full line is the average concentration for $x>h$ and the dashed line the concentration at $x=0.75 H$ from the symbolical computation. The triangles are the corresponding computed values with the numerical computation, where a grid with 100 points per phase is considered.

a measure of how close the exiting stream is to equilibrium. The overall residence time $t_{\text {res }}$ is defined as

$$
t_{\mathrm{res}}=\frac{W H L}{Q_{1}+Q_{2}} .
$$

For a given length $L$, a unique residence time and an efficiency $E(L)$ is obtained. $E=1$ corresponds to the situation when the exiting streams are in equilibrium and no further separation can take place.

The second characteristic which can describe the system is the extraction ratio $E_{r}$. It represents the fraction of the amount of solute that has been fed to the system which is removed by the second fluid. This is defined as

$$
E_{r}=\frac{1}{C^{\text {in }} Q_{1}} \int_{0}^{W} \int_{h}^{H} v_{2}(x, z) C_{2}(x, L, z) \mathrm{d} x \mathrm{~d} z
$$

Note that

$$
E=E_{r}\left(K \frac{Q_{1}}{Q_{2}}+1\right) .
$$

\subsection{Model validation}

The models developed are used to calculate the mass transfer characteristics under stratified flow conditions. The numerical algorithm proposed is validated with the symbolic solution for the plug flow regime. The simulation was performed for $D_{1}=D_{2}=7.410^{-9} \mathrm{~m}^{2} / \mathrm{s} ; v_{1}=v_{2}=0.0714$ $\mathrm{m} / \mathrm{s}, K=4.14$. For the symbolic solution the number of terms taken in the series expansion is 50 while the number of grid points taken in the transverse direction to the flow is 100 in each of the fluids for the numerical calculation. The concentration profiles obtained using the two approaches are shown in Fig. 4. Here a concentration at a transverse location and also the average concentration in the second fluid is shown. It is seen that the predictions of the two approaches are in perfect agreement.

As a further validation, the results of the $1 \mathrm{D}$ laminar flow should be approached by the $2 \mathrm{D}$ laminar flow by increasing the width of the channel. In Fig. 5 we see how increasing the width $W$ results in the $2 \mathrm{D}$ efficiency profiles converging to the $1 \mathrm{D}$ profile. It is seen that the ratio of $W / H$ should be around 25 for the $1 \mathrm{D}$ approximation to hold. Qualitatively the trends in the behaviour predicted by the $1 \mathrm{D}$ and $2 \mathrm{D}$ models always coincide. However for comparison of model predictions with actual micro-channel experiments the $2 \mathrm{D}$ numerical approximation must be used. This is necessary as the computed interface position $h$ is very different between the two approaches for 


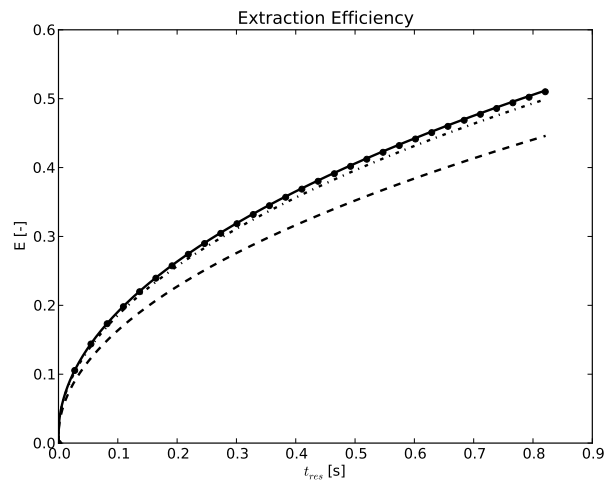

Figure 5: Convergence of the 2D efficiency to the 1D case. Full line is solution with the $1 \mathrm{D}$ model with $H=410^{-4}$ $\mathrm{m}, \mu_{1}=0.001025 \mathrm{~kg} / \mathrm{m} / \mathrm{s}=\mu_{2} / 2, Q_{1}=2 Q_{2}=14.286 \times 10^{-6} \mathrm{~m}^{2} / \mathrm{s}, K=4.14, D_{1}=2 D_{2}=7.410^{-9} \mathrm{~m}^{2} / \mathrm{s}$. For $2 \mathrm{D}$, dashed line, dash-dot, and dotted line are $W=10^{-4}, 10^{-3}, 10^{-2} \mathrm{~m}$ respectively, and with $Q_{1}=W \times 14.286 \times 10^{-6}$ $\mathrm{m}^{3} / \mathrm{s}$.

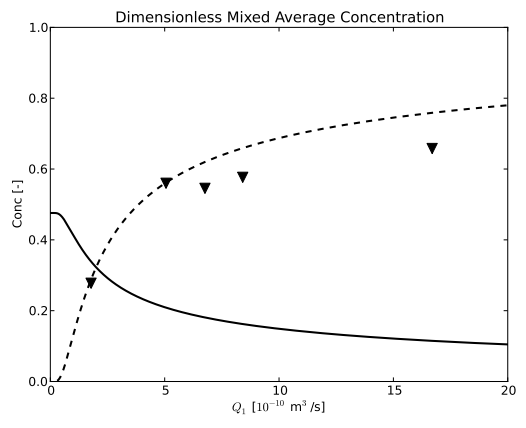

(a)

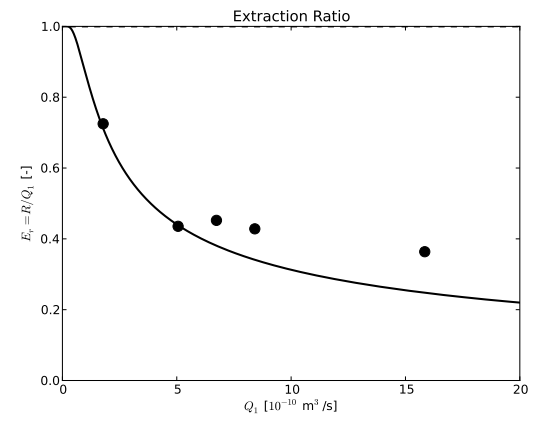

(b)

Figure 6: Comparison of (a) the dimensionless mixed cup concentration, dashed line aqueous phase, solid line organic phase; (b) extraction ratio, with the experimental results given in Žnidaršič-Plazl and Plazl [14] as symbols.

small widths and can significantly affect the quantitative results. For example, for the curves in Fig. 5 we have $h=0.526 H, 0.576 H$, and $0.579 H$ respectively as we increase $W$, while the $1 \mathrm{D}$ case gives $h=0.580 H$.

Furthermore, all numerical simulations were checked to verify the conservation of mass.

\subsection{Comparison with experimental results}

To the best of our knowledge we are not aware of any studies where extraction has been studied in the presence of electro-osmotic flow. The results from experimental studies under laminar flow conditions are hence used for validating the model predictions. As we will show, the predictions of the model compare favorably with the experimental results. In the next section we use the model to investigate if the plug flow conditions (electro-osmotic flow) can give better mass transfer performance as compared to the laminar flow conditions.

\subsubsection{Comparison with Žnidaršič-Plazl et al}

We first validate the numerical code with the behavior of a channel with a rectangular crosssection. The predictions of the model are compared with experimental results of Žnidaršič-Plazl and Plazl [14] in Fig. 6. Here we depict the variation of the dimensionless mixed average concentration and the extraction ratio on the flow-rate. There is a good agreement between the experimental data and the model predictions, even though the model is based on a simplified geometry and assumes fully developed flow. For the higher flow rates (and hence low residence 


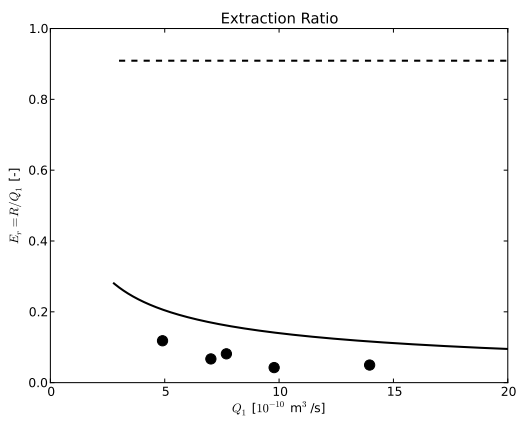

Figure 7: Comparison of our model predictions of the extraction ratio with the experimental results given in Okubo et al. [11] as symbols.

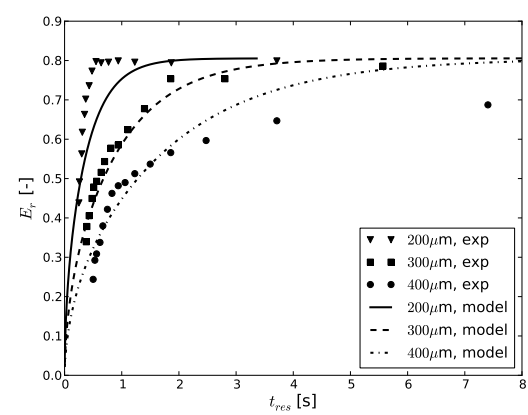

(a)

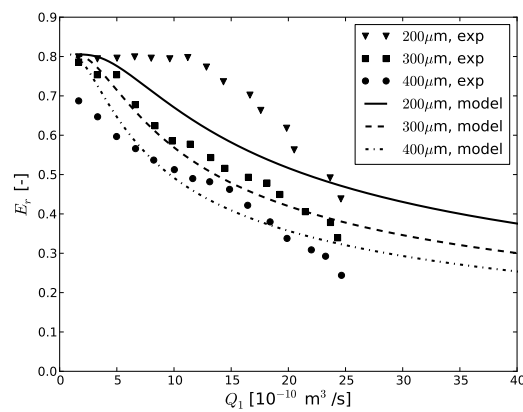

(b)

Figure 8: Comparison of our model predictions of the extraction ratio versus residence time and flow rate with the experimental results given in Fries et al. [6] as symbols.

times), the model underestimates the extraction ratio (the outlet concentration of $C_{1}$ is lower than that predicted by the model).

\subsubsection{Comparison with Okubo et al}

A comparison of our model predictions with the experimental results of Okubo et al. [11], is shown in Fig. 7. Here the horizontal dashed line shows the extraction ratio at equilibrium. The agreement of our model is similar to that obtained by the model presented in Okubo et al. [11]. We notice that now the model overestimates the experimental results for the flow rates used. In this experiment the total length of the micro-channel is short ( a straight channel of $33 \mathrm{~mm}$ is used as opposed to the $332 \mathrm{~mm}$ zigzag channel in Žnidaršič-Plazl and Plazl [14]). Consequently the residence times are low and equilibrium is not reached and the performance of the system is far from the equilibrium conditions.

\subsubsection{Comparison with Fries et al}

In Fig. 8 we compare the predictions of the model with the experimental results of Fries et al. [6]. The same experiment was analysed in Malengier et al. [10], but is shown here to allow comparison with the previous two experiments. Therefore, the figure shows the extraction ratio versus residence time and flow rate. The model predictions agree reasonably well with the experimental data, both qualitatively as well as quantitatively for most conditions. There are some conditions under which there are deviations. The equilibrium extraction ratio is 0.8 , which is seen as a clear upper bound in the figure. Decreasing the channel width leads to an enhancement of the extraction efficiency, see Fig. 8(a). This is to be expected as now we have lowered the diffusional resistance for the species to go from one phase to another. The slope for small residence times 


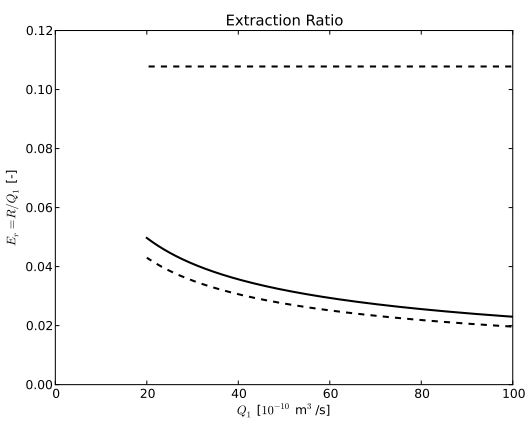

(a)

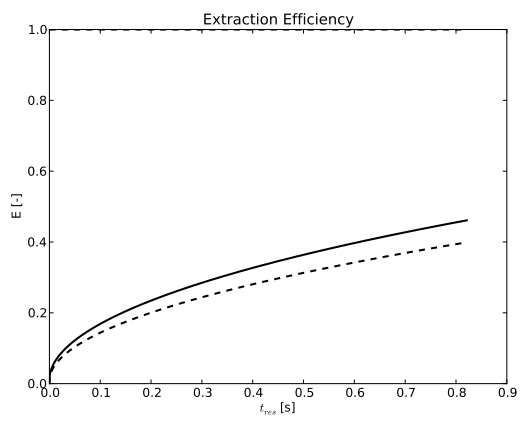

(b)

Figure 9: Comparison of 3D laminar flow (full line) and plug flow (dashed line) for $H=410^{-4} \mathrm{~m}, W=1.410^{-4}$ $\mathrm{m}, \mu_{1}=0.001025 \mathrm{~kg} / \mathrm{m} / \mathrm{s}=\mu_{2} / 2, Q_{1}=2 Q_{2}=14.28610^{-6} W \mathrm{~m}^{3} / \mathrm{s}, K=4.14, D_{1}=2 D_{2}=7.410^{-9} \mathrm{~m}^{2} / \mathrm{s}$. Left: extraction ratio, Right: extraction efficiency.

before achieving the equilibrium is the steepest for $W=200 \mu \mathrm{m}$ and decreases with increasing channel width. This means that for high throughput values, small changes of the volumetric flow rate result in a significant change of the extraction efficiency when the channel width is low.

The mismatch between the model predictions and the experimental results for the smallest channel $\left(6 \times 10^{-10}<Q_{1}<20 \times 10^{-10}\right)$ and for the largest channel for high residence time $\left(Q_{1}<7 \times 10^{-10}\right)$ can be attributed to several factors. First, no parameter fitting was done. In the simulations constant values for the diffusivity of vanillin in the two fluids is used. This has been estimated as $D_{\text {water }}=7.410^{-9}$ and $D_{\text {tol }}=5.6410^{-8} \mathrm{~m}^{2} / \mathrm{s}$. The experiments in the bigger $400 \mu \mathrm{m}$ channel reaches only an extraction ratio of 0.7 . We attribute this to the fact that the actual interface is farthest from the mid point for this dimension since in the experiments the two liquid streams were separated at the mid-point. For the $200 \mu \mathrm{m}$ channel, the diffusional resistance is lowered and equilibrium is attained faster experimentally as compared to the larger channels. The model predictions are in an overall sense consistent with the experimental measurements. This can be seen in the trends in the predicted values of $E_{r}$ which follow the trends of the experimental measurements. Specifically as we increase the size of the channel the time required for the same $E_{r}$ is more when the size of the channel is increased.

Having validated the model with experiments from the literature we now use it to analyse the effect of different parameters or operating conditions on mass transfer performance. Mathematical modelling is ideally suited for such an investigation and the insight it provides can be used to optimally design experiments.

\subsection{Plug flow versus Laminar flow}

It is well known that electro-osmosis can be used to modify flow-fields in micro-channels. Here an electric field induces an electro-osmotic effect and renders the flow more like an ideal plug flow where the dispersion effects are reduced. The effect of axial dispersion is normally thought of as one which reduces the efficiency of a process. To understand the role of dispersion a comparison is made between two cases one where the flow-field is laminar and the other where the flow of each liquid resembles an ideal plug flow. For a fair comparison the laminar flow is first simulated for a fixed value of individual flow-rates. In the simulation of the plug flow field the interface height is kept the same and the velocities of each of the phases is such that the flow rates are the same as in the laminar case. The velocities hence are the average of the profiles found in the laminar case. This ensures us that the residence time of each fluid as well as the overall residence time is matched for the two cases.

The behaviour of the laminar flow system is found to be superior to that of the PFR. For $Q_{1}=24.28 \times 10^{-6} \mathrm{~m}^{2} / \mathrm{s}$ and $Q_{2}=Q_{1} / 2, D_{1}=7.410^{-9} \mathrm{~m}^{2} / \mathrm{s}$ and $D_{2}=14.810^{-9} \mathrm{~m}^{2} / \mathrm{s}$ and $\mu_{1}=0.001 \mathrm{~kg} / \mathrm{m} / \mathrm{s}$ and $\mu_{2}=0.00025 \mathrm{~kg} / \mathrm{m} / \mathrm{s}$ the efficiency for the laminar flow field was found 


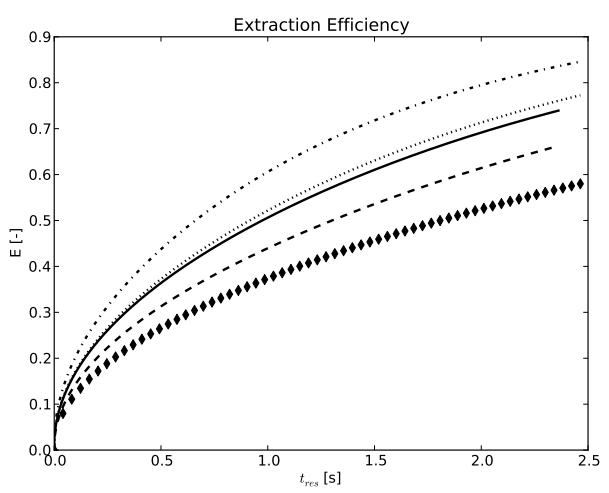

(a)

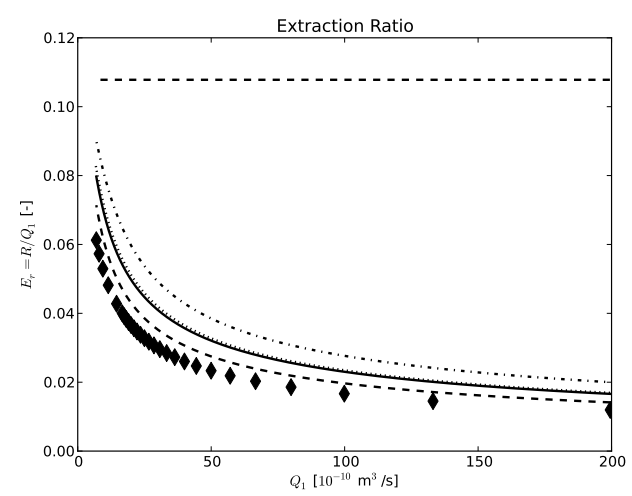

(b)

Figure 10: Comparison of the laminar flow versus PFR in terms of the interface height, fixed $Q_{1}$ and $Q_{2}$. Full line is laminar flow with $h=0.536 H$, dashed line PFR with same interface position. Diamond markers are PFR with $h=0.3 H$, dotted line PFR with $h=0.7 H$, and dash-dot line PFR with $h=0.8 H$.

to be 0.6838 and for the plug flow field was 0.5882 for the flat plate geometry. To confirm this the system was simulated for different flow-rate combinations such that the ratio was constant. This is equivalent to varying the residence time of the system. The results for this are shown in Fig. 9 for the rectangular micro-channel. Here it is seen that for all residence times the PFR performs worse than the laminar system as far as the extraction efficiency is concerned. The same trends were found for different parameter combinations.

This counter-intuitive result can be explained by looking at the magnitude of the velocity prevailing at the interface. In the plug flow situation the velocity magnitude is less than that in the laminar reactor. This arises since the laminar flow-field satisfies the no slip behaviour at the interface. The average velocity in the fluid is lower than that at the interface in the laminar flowfield in at least one of the phases (since the laminar flow-field has to satisfy the no-slip boundary condition). Consequently the mass transfer rate across the interface in the PFR is lower than that in the laminar flow regime and this gives rise to a deterioration in the performance in the equivalent plug flow system.

\subsection{Effect of interface position in plug flow}

In microfluidics it is possible to operate systems under a constant flow-rate or a constant pressure drop. In the case of pumps delivering a constant flow-rate, the imposition of an electric field to switch on the electro-osmotic effect would result in modulation of the flow-fields of the different phases. This in turn would mean that the height of the interface would change. To understand this behaviour numerical simulations have been performed by varying the height of the interface and assuming the velocity field to be a plug flow. When $h$ decreases keeping the flowrate $Q_{1}$ fixed the residence time of the first fluid decreases and that of the second fluid increases. This is expected to reduce the extraction efficiency as now the time spent by the first fluid in the micro-channel is less and the residence time of the second fluid increases. Both effects tend to make the efficiency poor.

Similarly it can be argued that when $h$ increases, $E$ becomes higher. The dependency of $E$ on residence times is shown in Fig. 10(a), and indeed shows the expected behaviour. As $h$ increases the performance in the plug flow exceeds that of the laminar flow. The same holds true for the extraction ratio, Fig. 10(b).

\section{Conclusions}

In this work the mass transfer behaviour in a stratified flow in a micro-channel has been analysed. The focus of the work is on determining the improvement in extraction when the flow 
field shifts from a laminar parabolic shape to a plug flow profile. The velocity and the concentration profiles were obtained symbolically for the plug flow system when the flow was between two flat parallel plates. The plug flow profile was found to perform worse than the laminar flow when the interface position and the residence times of each of the phases was kept the same. This is attributed to the lower velocity prevailing at the interface.

In this work we view electro-osmosis as a method to induce plug flow behavior. In the case of organic pollutants being extracted from an organic phase to an aqueous phase the effect of electric field on mass transfer will be minimal. The electric field modifies the flow-field from laminar to plug flow. The effect of this modification from laminar flow to plug flow is analysed in this work. The study shows clearly that the effect of electro-osmosis is beneficial only when the location of the interface height is modified in a particular direction by applying an electric field. It is detrimental otherwise.

It is shown that by controlling the interface height the plug flow profile can give a better performance than the laminar flow profile. But now the residence time of the fluids are different from that prevailing in the laminar flow. This shows that the effect of electro-osmosis can be beneficial only under certain operating conditions.

\section{Nomenclature}

\begin{tabular}{llll}
\hline Notation & Definition & Unit & Typical value \\
\hline$E$ & Extraction efficiency & - & $0-1$ \\
$E_{r}$ & Extraction ratio & - & $0-0.7$ \\
$H$ & Height of the micro-channel & $\mathrm{m}$ & $510^{-4}$ \\
$h$ & Interface position & $\mathrm{m}$ & $0.1 H-0.9 H$ \\
$K$ & Distribution ratio & - & $0.1-5$ \\
$L$ & Length of the micro-channel & $\mathrm{m}$ & $0.01-0.05$ \\
$t_{\text {res }}$ & Residence time & $\mathrm{s}$ & $1-10$ \\
$W$ & Width of the micro-channel & $\mathrm{m}$ & $10^{-4}$ \\
$\nabla P$ & Pressure gradient & $\mathrm{Pa} \mathrm{m}^{-1}$ & $1000-25000$ \\
$\mu_{1}, \mu_{2}$ & Viscosity in zone 1, 2 & $\mathrm{~kg} \mathrm{~m}^{-1} \mathrm{~s}^{-1}$ & $10^{-3}$ \\
$C_{1}, C_{2}$ & Concentration solute & $\mathrm{mol} \mathrm{m}^{-3}$ & $0.1-10$ \\
$D_{1}, D_{2}$ & Diffusion coefficient & $\mathrm{m}^{2} \mathrm{~s}^{-1}$ & $510^{-9}$ \\
$Q_{1}, Q_{2}$ & Flow rate & $\mathrm{m}^{2} \mathrm{~s}^{-1}(1 \mathrm{D})$ or & $10^{-6}$ \\
& & $\mathrm{~m}^{3} \mathrm{~s}^{-1}(2 \mathrm{D})$ & $10^{-9}$ \\
$v_{1}, v_{2}$ & Velocity & $\mathrm{m} \mathrm{s}^{-1}$ & 0.05 \\
\hline
\end{tabular}

\section{Acknowledgement}

The authors thank Roger Van Keer, coordinator of the research group $\mathrm{NaM}^{2}$, for his continuous interest and stimulation. The third author thanks the MaCKIE project (BOF-GOA 01GA0405) of Ghent University which made the collaboration of the 3 authors possible.

[1] Ahmed-Omer, B., Barrow, D., Wirth, T., 2008. Effect of segmented fluid flow, sonication and phase treansfer catalysis on biphasic reactions in capillary microreactors. Chemical Engineering Journal 135, S280-S283.

[2] Burns, J., Ramshaw, C., 1999. Development of micro-reactor for chemical production. Chemical Engineering Research and Design 1, 206-211.

[3] Burns, J., Ramshaw, C., 2001. The intensification of rapid reactions in multiphase systems using slug flow in capillaries. Lab on a Chip 1, 10-15.

[4] Cherlo, S., Kariveti, S., Pushpavanam, S., 2010. Experimental and numerical investigations of two-phase (liquid-liquid) flow behavior in rectangular microchannels. Industrial and Engineering Chemistry Research 49 (2), 893-899. 
[5] Dessimoz, A.-L., Cavin, L., Renken, A., Kiwi-Minsker, L., 2008. Liquid-liquid two-phase flow patterns and mass transfer characteristics in rectangular micro-reactors. Chemical Engineering Science 63 (16), 4035-4044.

[6] Fries, D., Voitl, T., von Rohr, P., 2008. Liquid extraction of vanillin in rectangular microreactors. Chemical Engineering \& Technology 31 (8), 1182-1187.

[7] Gao, Y., Wong, T. N., Yang, C., Ooi, K. T., 2005. Two fluid electroosmotic flow in microchannels. Journal of Colloid and Interface Science 284, 306-314.

[8] Guo, J., Ho, C., 2008. Theoretical study on membrane extraction of $\mathrm{Cu}^{2+}$ with D2EHPA in laminar flow circular tube modules. Desalination 233, 247-257.

[9] Liu, M., Liu, Y., Guo, Q., Yang, J., 2009. Modeling of electroosmotic pumping of nonconducting liquids and bio fluids by a two-phase flow method. Journal of Electroanalytical Chemistry $636,86-92$.

[10] Malengier, B., Pushpavanam, S., D’haeyer, S., 2011. Optimising performance of liquid-liquid extraction in stratified flow in micro-channels. J. Micromech. Microeng. 21, 13 pages.

[11] Okubo, Y., Maki, T., Aoki, N., Khoo, T. H., Ohmukai, Y., Mae, K., 2008. Liquid-liquid extraction for efficient synthesis and separation by utilizing micro spaces. Chemical Engineering Science 63 (16), 4070-4077.

[12] Ramkrihsna, D., Amundson, N., 1974. Transport in composite materials: Reduction to a self-adjoint formalism. Chemical Engineering Science 29, 1457-1464.

[13] Taha, T., Cui, Z., 2004. Hydrodynamics of slug flow inside capillaries. Chemical Engineering Science 59, 1181-1190.

[14] Žnidaršič-Plazl, P., Plazl, I., 2007. Steroid extraction in a microchannel system-mathematical modelling and experiments. Lab on a Chip 7, 883-889.

[15] Wang, C., Gao, Y., Nguyen, N.-T., Wong, T., Yang, C., Ooi, K., 2005. Interface control of pressure-driven two-fluid flow in microchannels using electroosmosis. Journal of Micromechanics and Microengineering 15, 2289-2297.

[16] Zhao, Y., Chen, G., Yuan, Q., 2007. Liquid-liquid two-phase mass transfer in the t-junction microchannels. A.I.Ch.E Journal 53, 3042-3053. 